\title{
Effect of Water Content on P-Wave Attenuation Across a Rock Fracture Filled with Granular Materials
}

\author{
Wei Wu $\cdot$ Jian Zhao
}

Received: 14 January 2014/Accepted: 8 May 2014/Published online: 30 May 2014

(C) Springer-Verlag Wien 2014

Keywords Rock fracture - Granular materials · Water content $\cdot$ P-wave attenuation

\section{Introduction}

Rock fractures are found to be ubiquitous in rock masses. Granular materials may exist in all scales of rock fractures, not only influencing seismic wave attenuation (Wu et al. 2013a), but also controlling rock mass instability ( Wu and Zhao 2014). Many recent studies consider air-dry granular materials sandwiched in a rock fracture and have found that the dynamic compaction of granular materials significantly affects P-wave attenuation across the filled fracture (Wu et al. 2012, 2013b, c). The water effect on P-wave attenuation across a filled rock fracture has received less attention. However, water or other liquid commonly exists in geological media and strongly influences the physical and mechanical properties of granular materials (Jaeger and Nagel 1996; Zheng and Spiers 2005). Water in granular materials induces cohesion between grains, lubricates grain friction, and causes additional energy dissipation (Mitarai and Nori 2006). Li et al. (2010) showed that the wave transmission coefficient decreases with higher water content in an unsaturated filled fracture. Ma et al. (2011) then used a three-phase medium model to analyze wave

W. Wu $(\bowtie)$

Laboratory of Rock Mechanics (LMR), School of Architecture, Civil and Environmental Engineering, École Polytechnique Fédérale de Lausanne (EPFL), 1015 Lausanne, Switzerland e-mail: wei.wu@epfl.ch

\section{J. Zhao}

Department of Civil Engineering, Monash University, Melbourne, VIC 3800, Australia transmission across the filled fracture. Nevertheless, the question remains open to understand how the change of water content physically affects the mechanical behaviors of granular materials and further influences P-wave attenuation across a filled fracture.

Recent studies in rock mechanics consider P-wave attenuation across a rock fracture through the description of boundary conditions (Pyrak-Nolte et al. 1990; Rokhlin and Wang 1991; Zhao and Cai 2001; Li et al. 2013). PyrakNolte et al. (1990) and Rokhlin and Wang (1991) introduced viscosity to the description of boundary conditions of a thin viscoelastic layer which may be used to estimate the water effect on wave attenuation across a filled fracture. However, not only viscosity but other water-induced factors, such as cohesion, lubrication and wave scattering, affect the attenuation. For P-wave attenuation, as less friction is induced in filling materials, wave scattering may play a more important role in the attenuation than viscosity. Hence, the previous works are based on simple situations, which may be insufficient to quantitatively predict P-wave attenuation across a filled fracture with different water contents. A recent focal point of studies on granular mechanics revealed that stress chains exist in granular materials to support external stresses (Majmudar and Behringer 2005). The grain contacts alter from asperity contacts in a dry state to capillary bridges in unsaturated and saturated states (Herminghaus 2005). Ponson et al. (2010) investigated propagation and scattering of nonlinear waves in disordered granular chains and found that wave decay depends on the disorder level in a chain. These studies can provide a physical explanation for $\mathrm{P}$-wave attenuation across a filled fracture.

The objectives of this study are to investigate the effect of water content on P-wave attenuation across a filled fracture from an air-dry state to a saturated state and to 
provide a qualitative explanation for the water effect on P-wave attenuation across the filled fracture. A wave transmission model is applied to interpret the experimental results and to reveal the physical change of granular materials with higher water content.

\section{Experimental Work}

The split Hopkinson rock bar (SHRB) method has been used to investigate $\mathrm{P}$-wave attenuation across a single rock fracture ( $\mathrm{Li}$ and $\mathrm{Ma}$ 2009; Wu et al. 2014) and a set of parallel rock fractures (Wu et al. 2013a, c). As shown in Fig. 1a, the rock bar system is similar to a conventional split Hopkinson pressure bar system and consists of a loading system with a norite striker bar, a pair of norite long bars, and a data acquisition unit. A compressed spring (stiffness $9.52 \mathrm{~N} / \mathrm{mm}$ ) was used as the energy source and launched the striker bar to impact the front end of the incident bar. A rubber disc was placed at the front end of the incident bar to protect the contact ends of the striker and incident bars and to generate a sine wave at a low loading rate. The impact event between the striker and incident bars generated a positive wave, which propagated along the loading direction. The positive wave was reflected at the bar end and propagated opposite to the loading direction as a negative wave. The P-wave across the filled fracture acted as a positive wave in the transmitted bar, and then was reflected at the bar end as a negative wave. If the length of two bars was smaller than the P-wave wavelength, the recorded P-wave would be superposed by the positive and negative waves. The $\mathrm{P}$-wave recorded from two strain gauge groups placed on each bar can be separated to obtain a positive wave and a negative wave. The $\mathrm{P}$-wave velocity in the norite material was $6,000 \mathrm{~m} / \mathrm{s}$; the frequency was about $2 \mathrm{kHz}$, and the amplitude was about 120 microstrain. The data acquisition unit measured the $\mathrm{P}$-wave through four strain gauge groups with a sampling rate of $100 \mathrm{kHz}$. More details about this method can be obtained from $\mathrm{Wu}$ (2013).

Quartz sand (material density $2,620 \mathrm{~kg} / \mathrm{m}^{3}$ ) was used as the filling material in this study. At the beginning, the quartz sand was sieved into particle sizes between 1 and $2 \mathrm{~mm}$. For an air-dry filled fracture, a layer of quartz sand was directly placed in a $2 \mathrm{~mm}$ pre-set gap between the incident and transmitted bars and was held by an aluminum
Fig. 1 a Schematic view of the SHRB method, b simulation of dry and unsaturated rock fractures, and $\mathbf{c}$ simulation of a saturated fracture (unit: $\mathrm{mm}$ )
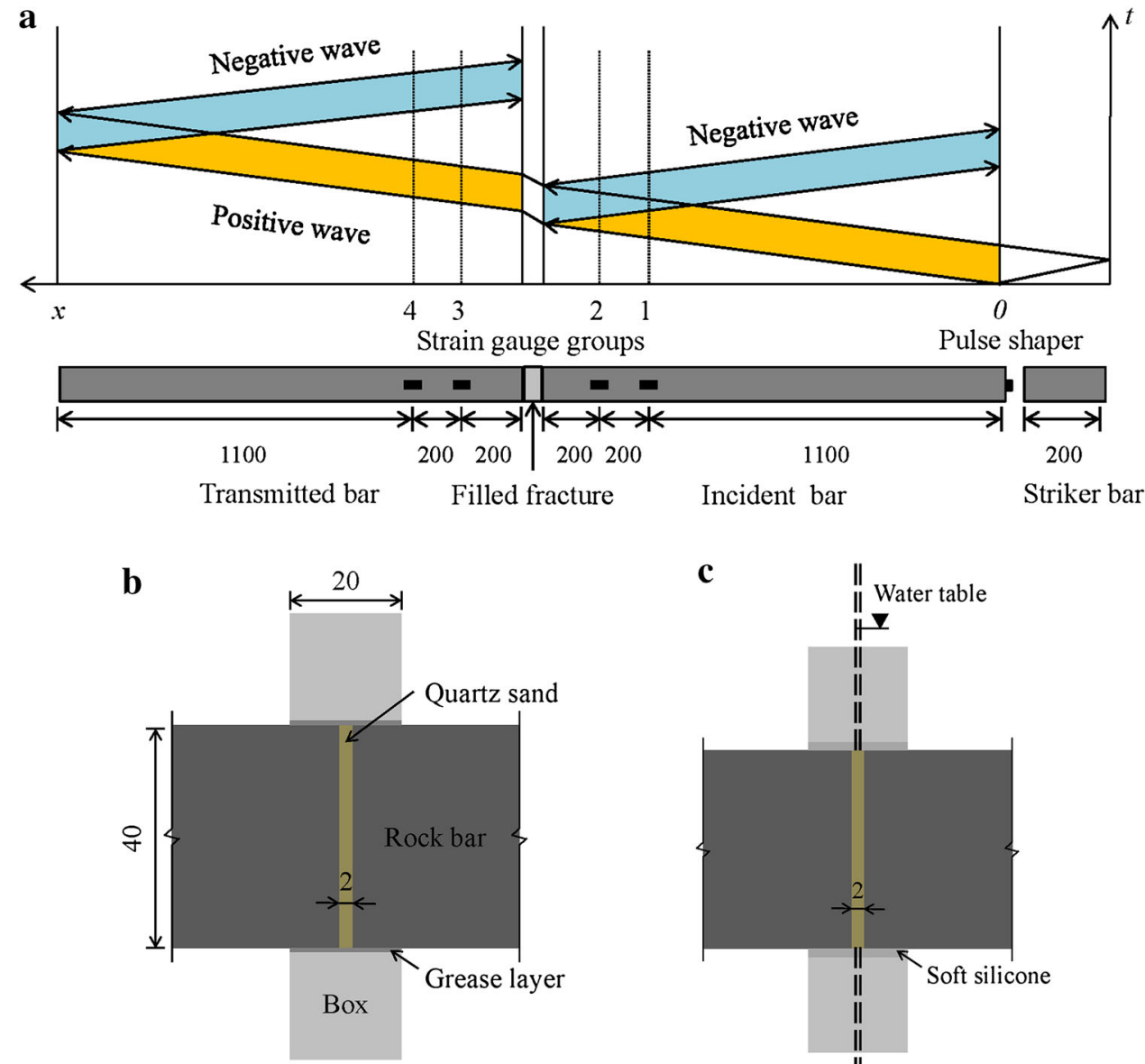
box (Fig. 1b). The gap between the bar surface and the confining box was filled by a $1 \mathrm{~mm}$ grease layer to minimize undesired friction. For an unsaturated filled fracture, the sand was first mixed with a designated amount of pure water, placed in the gap, and then held by the box. For a saturated filled fracture, a dry filled fracture was prepared first. The water was then injected into the sand layer through a small hole in the lower part of the box (Fig. 1c). When the bubble flow from the upper part of the box stopped, the filled fracture was considered to be in the saturated state. The gap between the bar surface and the box was connected by a $2 \mathrm{~mm}$ layer of soft silicone, which restricted water leakage from lateral sides of the box and allowed axial movement of the bars. The initial state of the filled fracture was uncompacted.

According to a previous study (Wu et al. 2013b), the porosity of quartz sand in a filled fracture was 0.397 , and the specific gravity of sand was 2.62 . Hence, the water content in a saturated filled fracture was equal to the porosity divided by the specific gravity, $15.15 \%$. Besides an air-dry filled fracture and a saturated filled fracture, we also considered an unsaturated filled fracture with three different water contents (i.e., 2, 5, and $10 \%$ ). Five SHRB tests were conducted for each case. If the amplitudes of transmitted waves from five tests were very close, a similar filling state of sand before each test would be guaranteed, and the sand would touch both sides of the fracture interfaces.

\section{Experimental Results}

The P-wave directly recorded by four strain gauge groups in the SHRB test on a saturated filled fracture is plotted in Fig. 2. The P-wave is normalized to the first P-wave amplitude recorded by strain gauge group 1 . The amplitude decrease of the second P-wave recorded by the strain gauge group 2 is due to the superposition of the positive and negative waves in the incident bar. The amplitudes of the P-wave recorded by strain gauge groups 3 and 4 are significantly reduced in the transmitted bar, owing to the dynamic compaction of the filling material. $\mathrm{Wu}$ et al. (2013b) addressed that the filling material delays the arrival time of the P-wave at the rear interface of the filled fracture and consumes a considerable amount of incident energy. This study additionally discusses the effect of water content on P-wave attenuation across a filled fracture.

The recorded $\mathrm{P}$-wave in two bars is superposed by a positive wave and a negative wave. Based on the wave separation calculation (Zhao and Gary 1997), the positive and negative waves at fracture interfaces are shown in Fig. 3. When the positive wave in the incident bar (considered as an incident wave) reaches the front interface of the filled fracture, the negative wave (considered as a

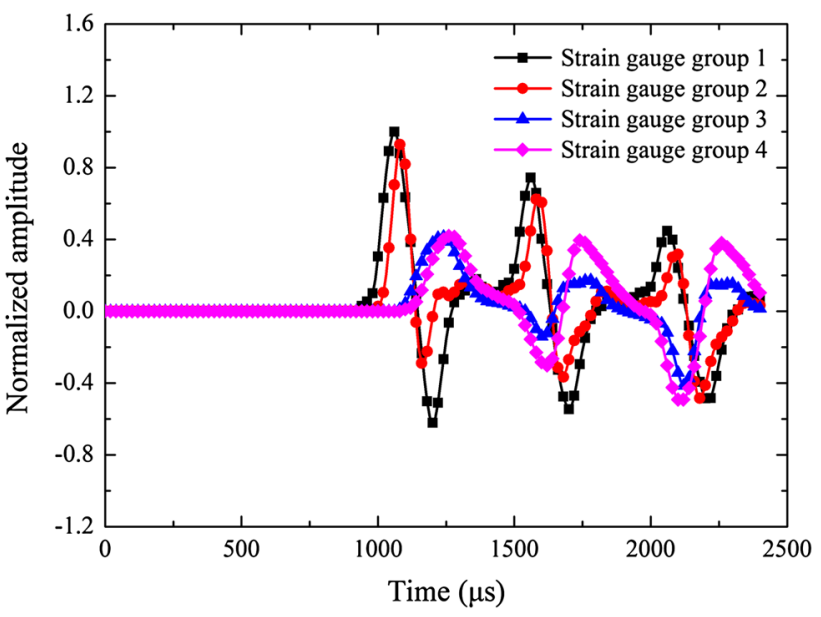

Fig. 2 Recorded P-wave from strain gauge groups in the SHRB test on a saturated filled fracture

reflected wave) is immediately generated and propagates opposite to the direction of the positive wave. After a time delay $(\Delta 1)$, owing to sand compaction, the positive wave at the fracture rear interface is initiated. The positive wave is then reflected at the free end of the transmitted bar as a negative wave, which reaches the rear interface after a time duration of $\Delta 2$. The positive wave in the transmitted bar is thus considered as a transmitted wave without the effect of a negative wave during the first wave loading. The attenuation factor is defined based on the positive wave amplitude in the incident bar, $\varepsilon_{\mathrm{PI}}$, and the positive wave amplitude in the transmitted bar, $\varepsilon_{\mathrm{PT}}$.

$1 / Q=\frac{1-\varepsilon_{\mathrm{PT}} / \varepsilon_{\mathrm{PI}}}{\pi}$

The effect of water content on P-wave attenuation across a filled fracture can be seen by comparing the incident wave and the transmitted waves from a filled fracture with different water contents (Fig. 4). The incident and transmitted waves in each case are also normalized to the $\mathrm{P}$-wave amplitude recorded by strain gauge group 1 . It can be observed that (1) the P-wave takes a longer time to reach the rear interface of a filled fracture with higher water content, and (2) the amplitude and the energy transmission rate (Wu et al. 2013a) decrease with higher water content in a filled fracture. This reveals that the water content plays a strong role in $\mathrm{P}$-wave attenuation across a filled fracture.

The P-wave attenuation across a filled fracture as a function of water content is demonstrated in Fig. 5. In general, the attenuation factor increases with higher water content. The augmentation of the attenuation factor quickly increases with higher water content when the filling material is close to an air-dry state, while the augmentation exhibits a slow increase when the filling material reaches a saturated state. This trend may be described by an exponential function as discussed below. 
Fig. 3 Positive and negative waves at fracture front and rear interfaces

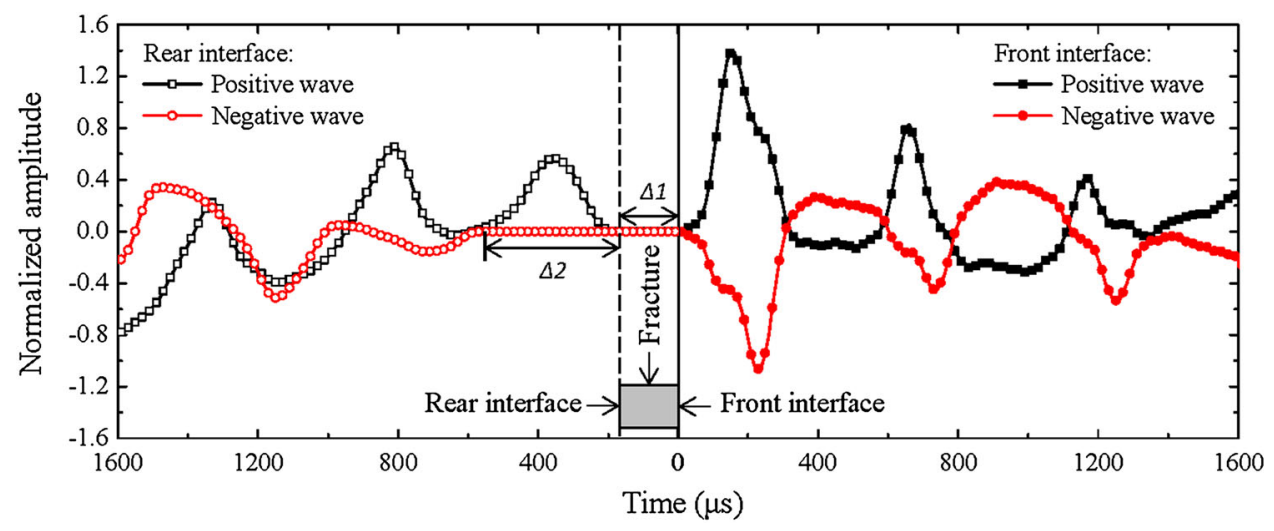

Figure 5 also shows the experimental results from a previous study ( $\mathrm{Li}$ et al. 2010). In that study, a filled fracture was sandwiched between a pair of cored granitic bars (length: about $1 \mathrm{~m}$ ), which were connected by a plastic tube. A generated P-wave was thus multiply reflected in the bar system to estimate the water effect on P-wave attenuation across a filled fracture with different beach sand thicknesses (e.g., 3, 4, and $5 \mathrm{~mm}$ ). The attenuation factor increases with higher water content from a dry state to an unsaturated state. The present study investigates the change in attenuation factor from an air-dry state to a saturated state based on a single P-wave incidence. Although there are differences between the previous and current testing methods (for instance, rock media, filling materials, and loading conditions), a similar trend can be observed with higher water content for a given fracture thickness.

\section{Discussion}

An inhomogeneous contact network supporting external loads, known as stress chains, has been observed in dry

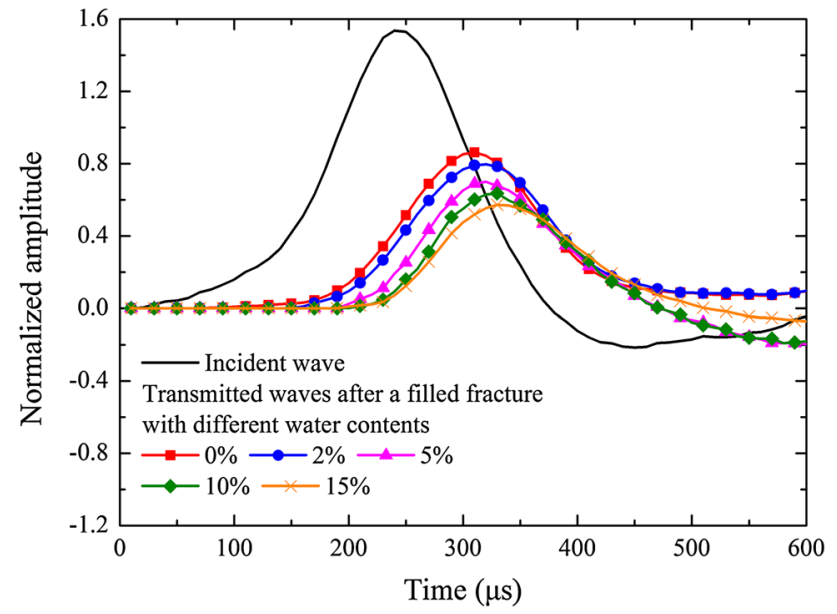

Fig. 4 An incident wave and transmitted waves from a filled fracture with different water contents granular materials (Majmudar and Behringer 2005). For wet granular materials, water strongly affects grain contacts and the mechanical behaviors of granular materials (Mitarai and Nori 2006). A wave transmission model can be used to describe the effect of water content on packed chains of sand grains (Ponson et al. 2010). In this model, a pair of contacting grains is considered as a diatomic unit, or a contact spin. When wave transmission exponentially decays with increasing disorder level, $D$, in stress chains, the ratio between the amplitude of a transmitted wave and that of an incident wave is $e^{-N D / \alpha}$. The attenuation factor can thus be expressed as

$1 / Q=\frac{1-e^{-N D / \alpha}}{\pi}$,

where the disorder level, $D=\left|N_{\text {up }}-N_{\text {down }}\right| /\left(N_{\text {up }}+\right.$ $\left.N_{\text {down }}\right)$, shows the amount of heterogeneity in granular materials. The total number of contact spin, $N$, is the sum of the number of contact spin with one orientation, $N_{\mathrm{up}}$, and the number of contact spin with the other orientation, $N_{\text {down }}$; the number of disordered spin, $N D$, is thus equal to

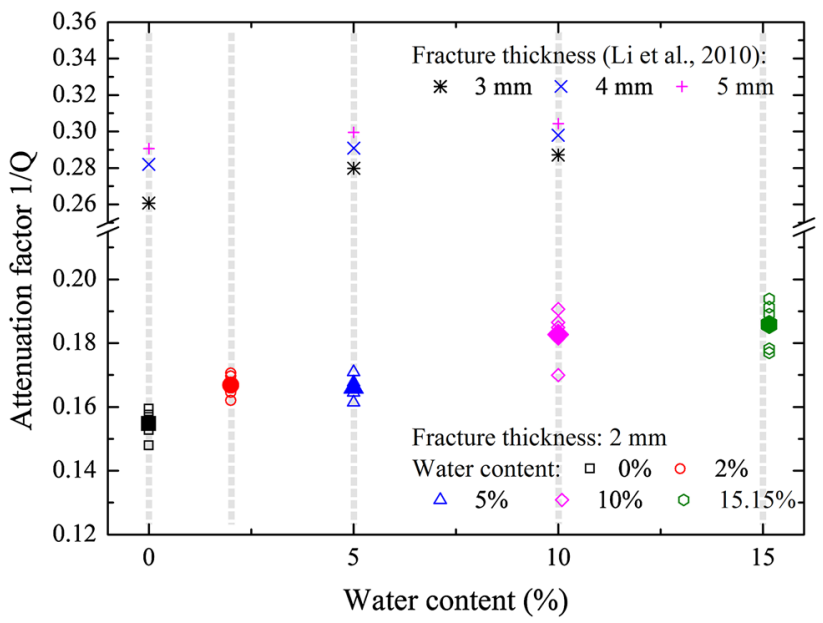

Fig. 5 Attenuation factor as a function of water content and comparison to previous results. The solid points indicate the average values 
twice the number of defects in stress chains, $N_{\text {defect }}$, which shows that the number of disordered spin increases with an increasing number of contact spins in an orientation opposite to wave propagation, and $\alpha$ is a constant value to describe grain geometry.

For a constant $\alpha$, the attenuation factor increases with an increasing number of disordered spins or defects in stress chains. Additionally, Herminghaus (2005) revealed that the defects influence the mechanical behaviors of granular materials with different water contents in two aspects. First, the amount of water negatively affects grain contacts during the formation of water bridges, because of suction pressure. Second, the incident energy is dissipated during the breaking of water bridges, owing to viscous stress. Hence, the water bridges in granular materials play a negative role in stress chains, which not only reduce grain contacts but induce energy dissipation. The number of water bridges increases with higher water content (Fournier et al. 2005), resulting in a greater number of defects and in a larger attenuation factor.

At the end point, we can explain two experimental observations in Fig. 4. (1) Because granular materials with higher water content contain a greater number of defects, it may take a longer time for the formation of stress chains. This phenomenon can also be observed in the study of Ponson et al. (2010). (2) A larger amount of incident energy is needed to break water bridges, owing to a larger number of water bridges existing in granular materials with higher water content. The amplitude of the transmitted wave thus decreases with higher water content in granular materials.

\section{Conclusions}

This experimental study shows that the attenuation factor in a filled fracture increases with higher water content from an air-dry state to a saturated state. According to a wave transmission model, it is found that a larger number of defects exists in granular materials with higher water content, which play a negative role in P-wave transmission and result in a larger attenuation factor. Based on the discussion on the physical change of granular materials with higher water content, not only viscosity, but grain contacts strongly affect the mechanical response of granular materials. This study suggests considering both factors in the future development of analytical predictions. The combination of the SHRB test on a filled fracture and the contact mechanics in granular media extends the knowledge regarding water's effect on P-wave attenuation across a rock fracture filled with granular materials; this is of importance in estimating the dynamic response of rock masses in engineering practices.
Acknowledgments This work was sponsored by the Swiss National Science Foundation (200020_147176).

\section{References}

Fournier A, Geromichalos D, Herminghaus S, Kohonen MM, Mugele F, Scheel M, Schulz M, Schulz B, SchierCh, Seemann R, Skudelny A (2005) Mechanical properties of wet granular materials. J Phys Condens Matter 17:S477-S502

Herminghaus S (2005) Dynamics of wet granular matter. Adv Phys $54: 221-261$

Jaeger HM, Nagel SR (1996) Granular solids, liquids, and gases. Rev Mod Phys 68:1259-1273

Li JC, Ma GW (2009) Experimental study of stress wave propagation across a filled rock joint. Int J Rock Mech Min Sci 46:471-478

Li JC, Ma GW, Huang X (2010) Analysis of wave propagation through a filled rock joint. Rock Mech Rock Eng 43:789-798

Li JC, Wu W, Li HB, Zhu JB, Zhao J (2013) A thin-layer interface model for wave propagation through filled rock joints. J Appl Geophys 91:31-38

Ma GW, Li JC, Zhao J (2011) Three-phase medium model for filled rock joint and interaction with stress waves. Int J Numer Anal Methods Geomech 35:97-110

Majmudar TS, Behringer RP (2005) Contact force measurements and stress-induced anisotropy in granular materials. Nature 435:1079-1082

Mitarai N, Nori F (2006) Wet granular materials. Adv Phys 55:1-45

Ponson L, Boechler N, Lai YM, Porter MA, Kevrekidis PG, Daraio C (2010) Nonlinear waves in disordered diatomic granular chains. Phys Rev E 82:021301

Pyrak-Nolte LJ, Myer LR, Cook NGW (1990) Transmission of seismic waves across single natural fractures. J Geophys Res 95:8617-8638

Rokhlin SI, Wang YJ (1991) Analysis of boundary conditions for elastic wave interaction with an interface between two solids. J Acoust Soc Am 89:503-515

Wu W (2013) Laboratory investigation of seismic response of rock fractures filled with granular materials. PhD thesis, Ecole Polytechnique Fédérale de Lausanne

Wu W, Zhao J (2014) A dynamic-induced direct-shear model for dynamic triggering of frictional slip on simulated granular gouges. Exp Mech 54:605-613

Wu W, Li JC, Zhao J (2012) Loading rate dependency of dynamic responses of rock joints at low loading rate. Rock Mech Rock Eng 45:421-426

Wu W, Zhu JB, Zhao J (2013a) A further study on seismic response of a set of parallel rock fractures filled with viscoelastic materials. Geophys J Int 192:671-675

Wu W, Zhu JB, Zhao J (2013b) Dynamic response of a rock fracture filled with viscoelastic materials. Eng Geol 160:1-7

Wu W, Li JC, Zhao J (2013c) Seismic response of adjacent parallel rock filled fractures with dissimilar properties. J Appl Geophys 96:33-37

Wu W, Li JC, Zhao J (2014) Role of filling materials in a P-wave interaction with a rock fracture. Eng Geol 172:77-84

Zhao J, Cai JG (2001) Transmission of elastic P-waves across single fractures with a nonlinear normal deformational behavior. Rock Mech Rock Eng 34:3-22

Zhao H, Gary G (1997) A new method for the separation of waves. Application to the SHPB technique for an unlimited duration of measurement. J Mech Phys Solids 45:1185-1202

Zheng X, Spiers CJ (2005) Compaction of granular calcite by pressure solution at room temperature and effects of pore fluid chemistry. Int J Rock Mech Min Sci 42:950-960 